\title{
Microwave single-scattering properties of randomly oriented soft-ice hydrometeors
}

\author{
D. Casella, A. Mugnai, P. Sanò, and M. Formenton \\ Istituto di Scienze dell'Atmosfera e del Clima, Consiglio Nazionale delle Ricerche, Roma, Italy \\ Received: 10 July 2008 - Revised: 20 July 2008 - Accepted: 24 September 2008 - Published: 14 November 2008
}

\begin{abstract}
Large ice hydrometeors are usually present in intense convective clouds and may significantly affect the upwelling radiances that are measured by satellite-borne microwave radiometers - especially, at millimeter-wavelength frequencies. Thus, interpretation of these measurements (e.g., for precipitation retrieval) requires knowledge of the single scattering properties of ice particles. On the other hand, shape and internal structure of these particles (especially, the larger ones) is very complex and variable, and therefore it is necessary to resort to simplifying assumptions in order to compute their single-scattering parameters.

In this study, we use the discrete dipole approximation (DDA) to compute the absorption and scattering efficiencies and the asymmetry factor of two kinds of quasi-spherical and non-homogeneous soft-ice particles in the frequency range 50-183 GHz. Particles of the first kind are modeled as quasispherical ice particles having randomly distributed spherical air inclusions. Particles of the second kind are modeled as random aggregates of ice spheres having random radii. In both cases, particle densities and dimensions are coherent with the snow hydrometeor category that is utilized by the University of Wisconsin - Non-hydrostatic Modeling System (UW-NMS) cloud-mesoscale model. Then, we compare our single-scattering results for randomly-oriented soft-ice hydrometeors with corresponding ones that make use of: a) effective-medium equivalent spheres, b) solid-ice equivalent spheres, and c) randomly-oriented aggregates of ice cylinders. Finally, we extend to our particles the scattering formulas that have been developed by other authors for randomlyoriented aggregates of ice cylinders.
\end{abstract}

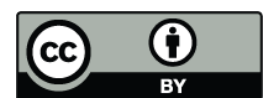

Correspondence to: D. Casella (daniele.casella@artov.isac.cnr.it)

\section{Introduction}

Several currently flown microwave radiometers provide observations in the frequency range $85-190 \mathrm{GHz}-$ e.g., the Special Sensor Microwave Temperature 2 (SSM/T2) and the Special Sensor Microwave Imager Sounder (SSMIS) onboard satellites of the US Defense Meteorological Satellite Program (DMSP), the Advanced Microwave Sounding Unit B (AMSU-B) aboard NOAA-15, 16, 17, 18 operational satellites, the Humidity Sounder for Brazil (HSB) aboard NASA's Aqua Mission, and the Microwave Humidity Sounder (HMS) onboard EUMETSAT's MetOp operational satellite. These microwave frequencies are highly responsive to scattering by frozen hydrometeors in precipitating clouds. Therefore, in order to use them for precipitation retrieval, it is necessary to understand and model the single-scattering properties (scattering, absorption, and extinction efficiencies; singlescattering albedo; and asymmetry factor) of the ice particles themselves (e.g. Gasiewski, 1992; Bennartz and Petty, 2001; Zhao and Weng, 2002; Bennartz and Bauer, 2003; Kongoli et al., 2003; Skofronick-Jackson et al., 2004; Hong et al., 2005a, b; Ferraro et al., 2005).

Because of the absence of direct measurements of the scattering properties of ice particles in precipitating clouds, one has to compute these parameters by means of Maxwell's electromagnetic equations. On the other hand, both in situ measurement field campaigns and laboratory experiments show that ice particles are usually non-homogeneous and non-spherical, and often have complex geometries being ice crystals of various habits (e.g. Bailey and Hallett, 2004; Heymsfield and Miloshevich, 2003). The discrete dipole approximation (DDA) originally developed by Draine and Flatau (1994) is a rigorous method that is capable of treating arbitrarily shaped and inhomogeneous particles. Therefore, it is recognized as the optimal technique to compute the

Published by Copernicus Publications on behalf of the European Geosciences Union. 
single-scattering properties of ice crystals and snow particles at microwave frequencies (e.g. Evans and Stephens, 1995; Liu, 2004; Kim, 2006).

However, the DDA method is computationally expensive to be applicable in remote sensing algorithms. Thus, simplified methods that make use of Mie theory and "equivalent" spheres are usually adopted. A simplified method that has been often used by the microwave remote sensing community for precipitation retrieval (e.g. Mugnai et al., 1990; Kummerow et al., 2001) approximates each non-spherical ice particle with a "fluffy sphere" (i.e., a homogeneous spherical mixture of ice and air) having the same mass than the ice particle and an equivalent volume $(V)$ which is usually taken as the volume of the circumscribing sphere. Noteworthy, a similar approximation has been also adopted for melting ice particles by using homogeneous spherical mixtures of ice, water and air (e.g. Meneghini and Liao, 1996; Bauer et al., 1999; Olson et al., 2001; Bennartz and Petty, 2001). The dielectric constants of these "equivalent" spheres are usually determined by effective-medium mixing theories, such as that of Maxwell Garnett (see Bohren and Huffman, 1983). In what follows, for simplicity we will refer to this method as Maxwell Garnett approximation.

Another simplified method that uses "equivalent" spheres was proposed by Grenfell and Warren (1999) (see also Neshyba et al., 2003) for the ultraviolet-visible-infrared portions of the electromagnetic spectrum, and then used for microwave radiation as well (e.g. Skofronick-Jackson et al., 2004). In this approximation, the single-scattering properties of each non-spherical ice particle are computed by means of a collection of equal-size solid-ice spheres having a diameter determined by the volume to cross-sectional area ratio (V/A) of the original non-spherical ice particle.

In this paper, we describe a new model that we have developed to represent quasi-spherical snow pellets and soft graupel particles. Then, we use the DDA method to compute the single-scattering parameters of these particles in the frequency range $50-183 \mathrm{GHz}$, and compare our results for randomly-oriented particles with corresponding results for the equivalent ice spheres of the Maxwell Garnett and Grenfell and Warren (1999) approximations, as well as with the scattering formulas developed by Weinman and Kim (2007) for randomly-oriented aggregates of ice cylinders. Finally, we extend these scattering formulas to our quasi-spherical soft-ice particles.

\section{Soft-ice particle models}

We have developed two different particle models (hereafter referred to as "kind A" and "kind B" particles) to represent quasi-spherical and non-homogeneous soft-ice particles, such as snow pellets and soft graupel. Kind A particles are modeled as quasi-spherical ice particles having randomly distributed spherical air inclusions, while kind B particles are

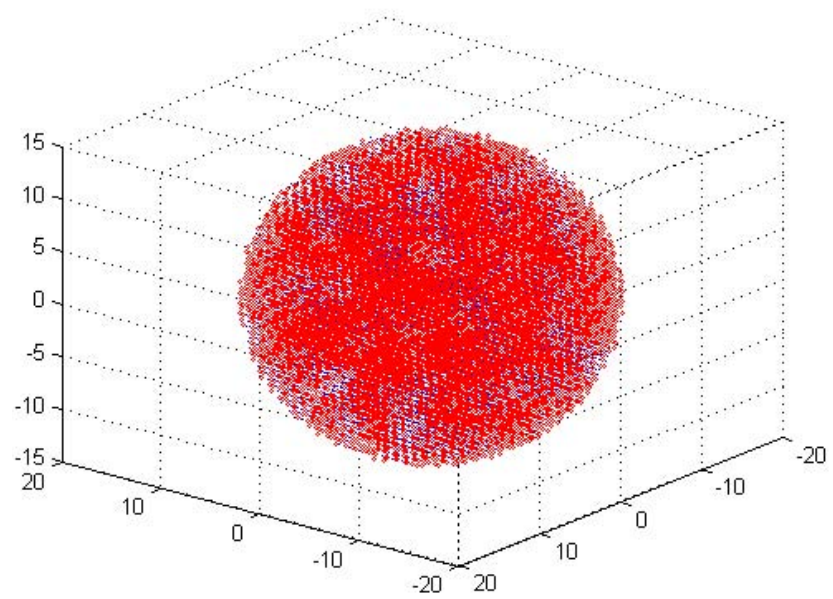

Fig. 1. Three-dimensional cartesian representation of a kind A quasi-spherical soft-ice particle with randomly-positioned pseudospherical air inclusions of random radii. Large red points represent solid-ice dipoles, while small blue points represent air dipoles. The coordinates are in units of the DDA lattice spacing $d$ (see Sect. 3 for more details), and the center of the circumscribing sphere is at $(0,0,0)$.

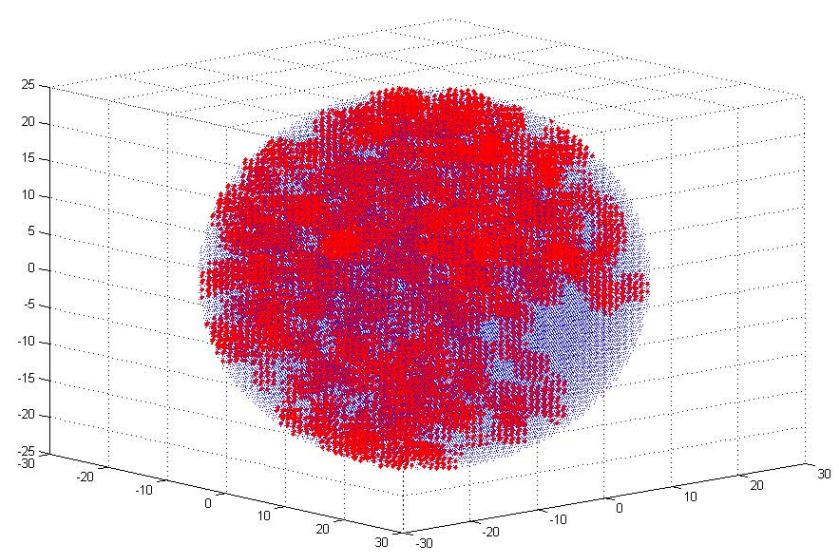

Fig. 2. Three-dimensional cartesian representation of a kind B quasi-spherical soft-ice particle made by randomly aggregating pseudo-spherical ice elements of random radii. As in previous Fig. 1, large red points represent solid-ice dipoles, small blue points represent air dipoles, and the coordinates are in units of the DDA lattice spacing $d$ (see Sect. 3 for more details) with the center of the circumscribing sphere at $(0,0,0)$.

modeled as quasi-spherical random aggregates of solid-ice spheres having random radii. Here, random means that a pseudorandom array in the code sets both the position of the center of every pseudo-spherical air inclusion (kind A) or of every pseudo-spherical ice component (kind B), and the radius of the pseudo-spherical inclusion/component itself. Examples of kind A and kind B particles are shown in Figs. 1 and 2 , respectively. 


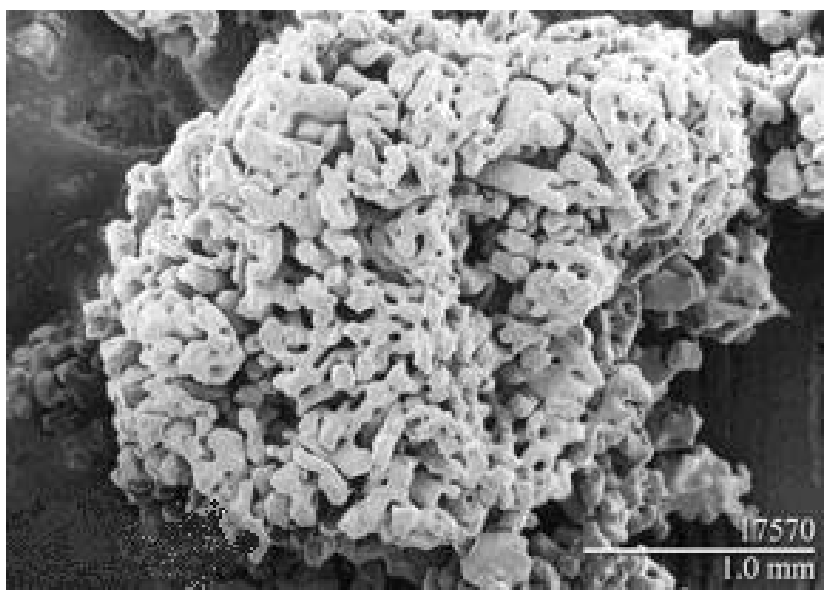

Fig. 3. Graupel particle as observed by a low temperature scanning electron microscope (LT-SEM). Courtesy of Electron and Confocal Microscopy Laboratory, Agricultural Research Service, US Department of Agriculture http://emu.arsusda.gov/snowsite/rimegraupel/.

While rimed ice particles can have different shapes, graupel particles can be either conical or oblate spheroidal or can have an apple shape, snow pellets are almost spheroidal (Pruppacher and Klett, 1997), in this preliminary study we have chosen to use quasi-spherical shapes. Nevertheless, comparison with Fig. 3 indicates that our model particles may be reasonable representations of soft-ice particles that are found in nature. Specifically, kind A and kind B particles may be used to simulate large graupel particles with air bubbles included, and small snow pellets with rimed drops that are still distinguishable, respectively.

In both models, particle bulk density and dimensions are coherent with the snow hydrometeor category that is utilized by the University of Wisconsin - Non-hydrostatic Modeling System (UW-NMS) cloud-mesoscale model developed by Tripoli (1992), which we use for generating the cloudradiation databases (Tripoli et al., 2007) for our precipitation retrieval algorithm (Sanò et al., 2008) - i.e., snow density $\left(\rho_{\text {snow }}\right)$ is fixed to $0.2 \mathrm{~g} / \mathrm{cm}^{3}$ and the radius $(r)$ of the circumscribing sphere varies from $0.013 \mathrm{~cm}$ to $0.5 \mathrm{~cm}$.

\section{Discrete dipole approximation method}

We use the discrete dipole approximation (DDA) developed by Draine and Flatau $(1994,2004)$ to compute the single scattering properties of our non-spherical soft-ice particles. The DDA method is a flexible technique which accurately computes the light scattering by arbitrarily shaped and inhomogeneous particles, and it has been used in various applications to study the scattering by dust and ice particles (e.g. Evans and Stephens, 1995; Liu, 2004; Evans et al., 2005; Kim, 2006). In the DDA method, the arbitrarily shaped target continuum is represented by an array of polarizable point dipoles. The scattering properties of the specific target - which include the absorption efficiency, scattering efficiency, extinction efficiency, and $4 \times 4$ Mueller scattering intensity matrix - are calculated by the interaction between the array of dipoles and a monochromatic plane wave incident from infinity.

The main advantage of the DDA method is that it is completely flexible regarding the geometry of the target. The point dipoles in the array representing the arbitrary target occupy the positions on a cubic lattice with a lattice spacing $d$ which is required to be small compared both to any structural lengths in the target and the incident wavelength $(\lambda)$. According to the authors, the DDA method gives adequately accurate results (i.e., errors in the single-scattering albedo less than 1\%) if $|m| k d<1$, where $m$ is the complex refractive index of the target material, and $k=2 \pi / \lambda$ is the wave number; in addition, the authors find that in order to compute the scattering phase function, a more conservative criterion $|m| k d<0.5$ is required. In this study, a variable dipole number $N\left(N=V / d^{3}\right.$, where $V$ is the volume of the target $)$ is used for the different particle sizes and frequencies, so as to ensure the more conservative criterion.

\section{Results}

We have computed the single-scattering quantities (scattering, absorption, and extinction efficiencies; single-scattering albedo, and asymmetry factor) for the two kinds of soft-ice model particles that are described in Sect. 2. Calculations were made at four microwave frequencies $(50,89,150$, and $183 \mathrm{GHz}$ ) for kind A particles, and only at $89 \mathrm{GHz}$ for kind B particles. All results that are shown in this Section are for random orientation and were obtained by averaging over 100 different particle orientations. In addition, we only show the scattering/extinction efficiencies and the asymmetry factor because absorption by ice is very low at these frequencies - absorption efficiency is usually less than 0.02 while scattering efficiency can be as large as 3.2.

As an example, Fig. 4 shows the scattering efficiency $\left(Q_{\text {sca }}\right.$ - defined here as the scattering cross section divided by the cross section of the circumscribing sphere) and the asymmetry factor $(g)$ at $89 \mathrm{GHz}$ for kind A and kind B particles having radii $(r)$ smaller than $0.5 \mathrm{~cm}$. For comparison, the corresponding results for two equivalent-sphere approximations (Maxwell Garnett and Grenfell and Warren, 1999) are also shown. Noteworthy, while the asymmetry factors for the two kinds (A and B) of quasi-spherical soft-ice particles are almost identical, noticeable differences are found for the scattering efficiency - especially above $0.4 \mathrm{~cm}$, where $Q_{\text {sca }}$ starts decreasing for kind $\mathrm{B}$ while keeps increasing for kind A.

It is evident that the Grenfell and Warren approximation does not adequately represent the scattering properties 

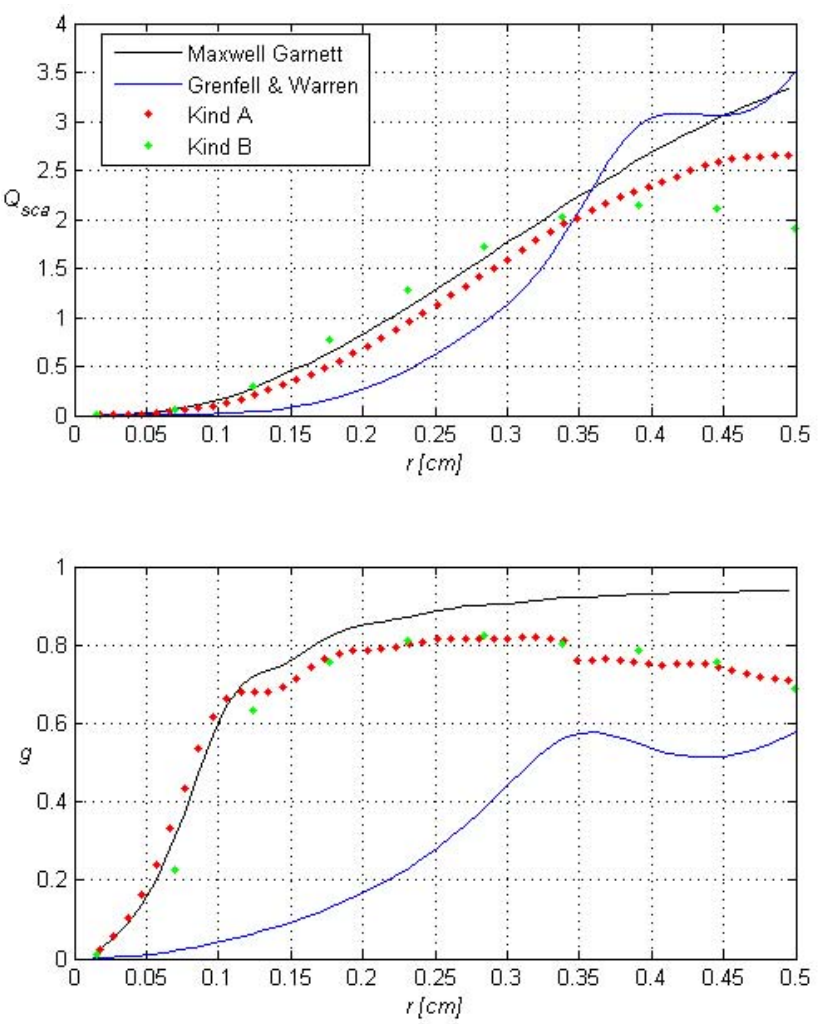

Fig. 4. Scattering efficiency ( $Q_{\text {sca }}-$ top panel) and asymmetry factor ( $g$ - bottom panel) at $89 \mathrm{GHz}$ as a function of radius of circumscribing sphere $(r)$ for randomly-oriented kind A (red dots) and kind $\mathrm{B}$ (green dots) particles having density equal to $0.2 \mathrm{~g} / \mathrm{cm}^{3}$. For comparison, corresponding results for Maxwell Garnett (black lines) and Grenfell and Warren (1999) (blue lines) equivalent spheres are also shown.

of quasi-spherical soft-ice particles - especially, because it greatly underestimates the asymmetry factor, while significantly underestimating/overestimating the scattering efficiency for particles smaller/larger than about $0.35 \mathrm{~cm}$. In contrast, the Maxwell Garnett approximation seems to be acceptable for particles smaller than about $0.3 \mathrm{~cm}$ (i.e., for Mie size parameters $x=2 \pi r / \lambda$ smaller than 5.5). Differences from "exact" scattering quantities are less than $10 \%$ at these sizes, but rapidly increase for larger sizes - in particular, while the "exact" asymmetry factor constantly decreases from about 0.8 to about 0.7 , it slightly increases from about 0.9 to about 0.93 for the Maxwell Garnett approximation.

Similar comments can be made for Fig. 5 which shows the single-scattering results at $150 \mathrm{GHz}$ for kind A particles having radii smaller than $0.5 \mathrm{~cm}$, as well as for corresponding Maxwell-Garnett and Grenfell and Warren (1999) equivalent spheres - the main difference with the previous figure being that the Maxwell Garnett approximation now seems to be acceptable up to about $0.15 \mathrm{~cm}$ (i.e., for Mie size parameters $x .<5$, which is equivalent to the range that was found for
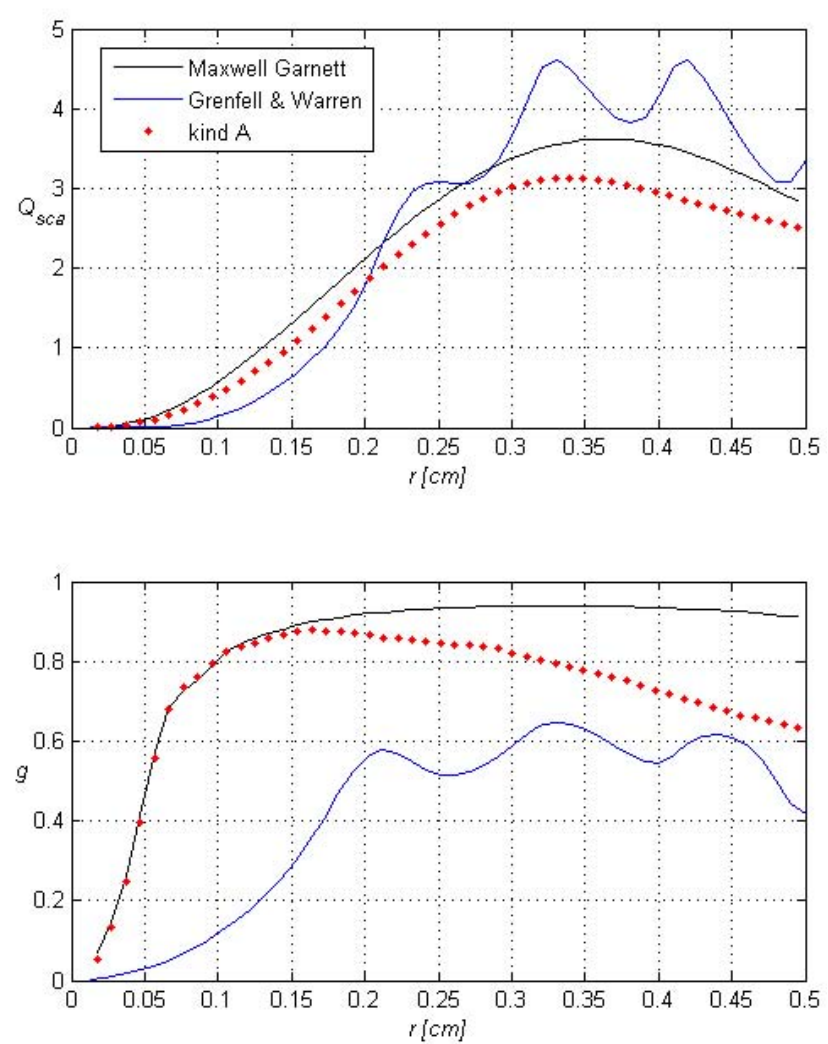

Fig. 5. Same as Fig. 4, but for kind A particles only and at $150 \mathrm{GHz}$.

$89 \mathrm{GHz}$ ). In addition, the scattering quantities of our soft-ice particles do not show any of the characteristic Mie oscillations that are evident for the solid-ice spheres of the Grenfell and Warren (1999) approximation.

The Maxwell Garnett approximation fails above $r \sim 0.15 \mathrm{~cm}$ as all differences tend to increase. In particular, this approximation significantly underestimates (up to more than $20 \%$ ) the scattering efficiency, while greatly overestimating the asymmetry factor - which is almost constant at about 0.9-0.95 for the Maxwell Garnett soft-ice spheres, while constantly decreasing from about 0.87 to about 0.62 for our soft-ice non-homogeneous particles. This latter result confirms an important conclusion of SkofronickJackson et al. (2004) who found in their attempt to retrieve snowfall over land from AMSU-B measurements, that using the Maxwell Garnett approximation to perform radiative transfer calculations would generate very large asymmetry factors $(>0.9)$ at the higher microwave frequencies and would not adequately "cool" the upwelling radiation, as compared to AMSU-B measurements.

By applying the anomalous diffraction theory, Weinman and Kim (2007) find the following fitting formulas to their DDA computations for randomly-oriented aggregates of ice cylinders: 
$\frac{Q_{\mathrm{ext}}(\rho)}{\rho}=\frac{0.40 \rho^{2.1}}{1+0.031 \rho^{4.1}}, \quad g=\frac{0.30 \rho^{2.1}}{1+0.18 \rho^{2.6}}$

where $Q_{\text {ext }}$ and $g$ are the extinction efficiency (defined as the extinction cross section divided by the particle projected area $A_{\perp}$ ) and the asymmetry factor, respectively, while $\rho$ is the phase delay suffered by the radiation as it passes through the particle. According to van de Hulst (1957), $\rho=k \Delta(m-1)$ where $\Delta$ is a length representing the effective dimension of the particle - for cylinders, $\Delta$ is given by (Weinman and Kim, 2007):

$\Delta=\frac{4 V}{\pi A_{\perp}}$

In order to compare our single-scattering DDA computations for soft-ice quasi-spherical particles with the Weinman and Kim (2007) fitting formulas, we have to compute the phase delay $\rho$ for our particles. To this end, we use the following definition of the effective dimension $\Delta$, which is appropriate for a mixture of spheres (van de Hulst, 1981, p. 194):

$\Delta=\frac{3}{2} \frac{V}{A_{\perp}}$

where $A_{\perp}$ and $V$ for our soft-ice particles are respectively given by the total projected area and by the total volume of the ice dipoles forming the particle:

$A_{\perp}=f \pi r^{2}, \quad V=\frac{\rho_{\text {snow }}}{\rho_{\text {ice }}} \frac{4}{3} \pi r^{3}$

Here, $r$ is the radius of the circumscribing sphere, $\rho_{\text {ice }}=0.916 \mathrm{~g} / \mathrm{cm}^{3}$ is the density of pure ice, and the factor $f$ has been computed by utilizing several different particle realizations in random orientation both for kind $\mathrm{A}$ and kind B particles - we find $f_{A}=0.95$ and $f_{B}=0.78$, respectively.

The comparison is presented in Fig. 6, which shows $Q_{\text {ext }} / \rho$ and $g$ from Weinman and Kim (2007) fitting formulas as a function of the phase delay $\rho$, together with the corresponding values from our DDA computations for kind A particles at four frequencies $(50,89,150$, and $183 \mathrm{GHz})$ and for kind B particles at $89 \mathrm{GHz}$ only. Evidently, Weinman and Kim (2007) fitting formulas do not adequately represent the scattering from our soft-ice particles. This is not unexpected due to the fact that these authors attempt to model snow flakes and therefore use complex non-spherical particle shapes that are very different from our quasi-spherical shapes. In particular, $Q_{\mathrm{ext}} / \rho$ from the fitting formulas shows a peak at about $\rho=2.4$ which is $20-30 \%$ higher than the highest values for our soft-ice particles - which are usually found at larger $\rho$ values (between 2.4 and 3.1). In addition, the fitting formula for the asymmetry factor largely underestimates the results for our soft-ice particles up to about $\rho \sim 3$.

On the other hand, the behavior of the fitting formulas is overall similar to that of our computations. Thus, we have modified the fitting parameters so as to best represent our results. We find:

$\frac{Q_{\mathrm{ext}}(\rho)}{\rho}=\frac{0.35 \rho^{1.53}}{1+0.023 \rho^{3.42}}, \quad g=\frac{2.54 \rho^{2.09}}{1+2.45 \rho^{2.33}}$
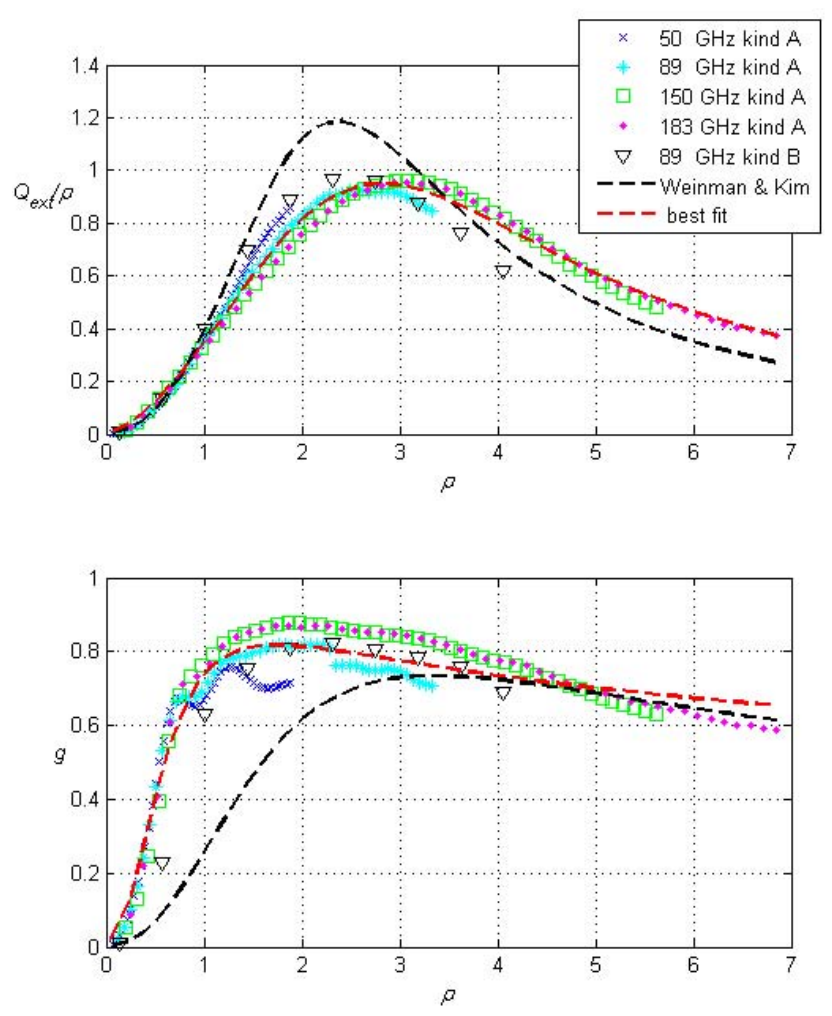

Fig. 6. Extinction efficiency divided by the phase delay ( $Q_{\text {ext }} / \rho-$ top panel) and asymmetry factor ( $g$ - bottom panel) as a function of phase delay $(\rho)$ for kind A particles at four frequencies $(50,89$, 150 , and $183 \mathrm{GHz}$ ) and for kind B particles at $89 \mathrm{GHz}$. For comparison, corresponding results from the fitting formulas developed by Weinman and Kim (2007) for randomly-oriented aggregates of ice cylinders (black dashed lines) and best-fit results obtained by modifying the fitting parameters of these formulas (red dashed lines).

The results from the modified fitting formulas are also shown in Fig. 6. Obviously, the new fitting formulas represent much better the scattering properties of both kind $\mathrm{A}$ and kind $\mathrm{B}$ particles - relative errors are usually less than $20 \%$. Thus, we plan to perform a sensitivity study on the accuracy of these formulas for computing the upwelling brightness temperatures that would be measured by space-borne microwave radiometers.

\section{Conclusions}

We have developed two different models (kind A and kind B) to represent quasi-spherical snow pellets and soft graupel particles, and have used the DDA method to compute the single-scattering parameters of these particles for particle density equal to $0.2 \mathrm{~g} / \mathrm{cm}^{3}$ and in the frequency range $50-183 \mathrm{GHz}$. Then, we have compared our results for randomly-oriented soft-ice particles with corresponding results for two kinds of equivalent ice spheres (referred to as 
Maxwell Garnett and Grenfell and Warren, 1999, equivalent spheres), as well as with the fitting formulas developed by Weinman and Kim (2007) for randomly-oriented aggregates of ice cylinders.

The main results are:

- Single-scattering results at $89 \mathrm{GHz}$ for kind A and kind B particles are quite similar - especially for the asymmetry factor. This is somewhat unexpected because as shown by Figs. 1 and 2, the two kinds of particles may look rather different. In future work, we plan to verify this result at different frequencies and/or for different particle densities.

- The Grenfell and Warren (1999) approximation doesn't ever adequately represent the scattering properties of our soft-ice particles. This is not surprising if we think that these authors attempt to model the scattering properties at ultraviolet-visible-infrared frequencies (not at microwave frequencies) of ice crystals having shapes that are very different from our quasi-spherical shapes.

- In contrast, the Maxwell Garnett approximation may give acceptable results depending on particle size and dimensions of the inclusions - a result which has been already pointed out by other authors (e.g. Bohren, 1985) who find that the optical dimensions of the inclusions should be $\ll 1$ for a correct application of the mixing theory. On the other hand, this approximation may produce unrealistically high values of the asymmetry factor for soft-ice particles, which, as demonstrated by Skofronik-Jackson et al. (2004), would lead to computed upwelling brightness temperatures that are inconsistent with the measurements.

- Even the Weinman and Kim (2007) fitting formulas do not adequately represent the scattering from our softice particles, but again this is not surprising since these authors model snow flakes rather than quasi-spherical shapes.

- Nevertheless, we have been able to modify these fitting formulas in such a way to obtain very good fits - differences from DDA results are usually less than $10-20 \%$.

Finally, we plan in our future work to: a) simulate more realistic rimed particles, with shells of different densities and conical or spheroidal shapes; and b) investigate the impact of our soft-ice particles on the microwave radiative transfer to space through intense convective clouds.

Acknowledgements. The authors would like to thank James Weinman for very useful comments and suggestions. This study has been supported by the European Commission FP6 programme through the project "Observation, Analysis and Modeling of Lightning Activity in Thunderstorms, for use in Short Term Forecasting of Flash Floods" (FLASH) and by the Italian FISR-MIUR programme "Sustainable Development and Climate Changes" through the project "Aerosol Effects on Clouds and Climate" (AEROCLOUDS).

Edited by: F. Guzzetti

Reviewed by: J. A. Weinman

\section{References}

Bailey, M. and Hallett, J.: Growth rates and habits of ice crystals between $-20^{\circ}$ and $-70^{\circ} \mathrm{C}$, J. Atmos. Sci., 61, 514-544, 2004.

Bauer, P., Baptista, J. P. V. P., and de Iulis, M.: The effect of the melting layer on the microwave emission of clouds over the ocean, J. Atmos. Sci., 56, 852-867, 1999.

Bennartz, R. and Petty, G. W.: The sensitivity of microwave remote sensing observations of precipitation to ice particle size distributions, J. Appl. Meteorol., 40, 345-364, 2001.

Bennartz, R. and Bauer, P.: Sensitivity of microwave radiances at $85-183 \mathrm{GHz}$ to precipitating ice particles, Radio Sci., 38(4), 8075, doi:10.1029/2002RS002626, 2003.

Bohren, C. F. and Huffman, D. R.: Absorption and Scattering of Light by Small Particles, Wiley-Interscience, New York, 530 pp., 1983.

Bohren, C. F.: Applicabilty of the effective-medium theories to problems of scattering and absorption by nonhomogeous atmospheric particles, J. Atmos. Sci., 43, 468-475, 1985.

Draine, B. T. and Flatau, P. J.: Discrete-dipole approximation for scattering calculations, J. Opt. Soc. Am. A, 11, 1491-1499, 1994.

Draine, B. T. and Flatau, P. J.: User Guide to the Discrete Dipole Approximation Code DDSCAT 6.1, http://arxiv.org/abs/ astro-ph/0409262v2, 2004.

Evans, K. F. and Stephens, G. L.: Microwave radiative transfer through clouds composed of realistically shaped ice crystals: Part I. Single scattering properties, J. Atmos. Sci., 52, 2041-2057, 1995.

Evans, K. F., Wang, J. R., Racette, P. E., Heymsfield, G., and Li, L.: Ice cloud retrievals and analysis with the Compact Scanning Submillimeter Imaging Radiometer and the Cloud Radar System during CRYSTAL FACE, J. Appl. Meteorol., 44, 839-859, 2005.

Ferraro, R. R., Weng, F., Grody, N. C., Zhao, L., Meng, H., Kongoli, C., Pellegrino, P., Qiu, S., and Dean, C.: NOAA operational hydrological products derived from the advanced microwave sounding unit, IEEE T. Geosci. Remote, 43, 1036-1049, 2005.

Gasiewski, A. J.: Numerical sensitivity analysis of passive EHF and SMMW channels to tropospheric water vapor, clouds, and precipitation, IEEE T. Geosci. Remote, 30, 859-870, 1992.

Grenfell, T. C. and Warren, S. G.: Representation of a nonspherical ice particle by a collection of independent spheres for scattering and absorption of radiation, J. Geophys. Res., 104, $31697-$ $31709,1999$.

Heymsfield, A. J. and Miloshevich, L. M.: Parameterization for the cross-sectional area and extinction of cirrus and stratiform ice cloud particles, J. Atmos. Sci., 60, 936-956, 2003.

Hong, G., Heygster, G., Miao, J., and Kunzi, K.: Detection of tropical deep convective clouds from AMSU-B water vapor channels measurements, J. Geophys. Res., 110, D05205, doi:10.1029/2004JD004949, 2005a. 
Hong, G., Heygster, G., Miao, J., and Kunzi, K.: Sensitivity of microwave brightness temperatures to hydrometeors in a tropical deep convective cloud system at $89-190 \mathrm{GHz}$, Radio Sci., 40, RS4003, doi:10.1029/2004RS003129, 2005b.

Kim, M.-J.: Single scattering parameters of randomly oriented snow particles at microwave frequencies, J. Geophys. Res., 111, D14201, doi:10.1029/2005JD006892, 2006.

Kongoli, C., Pellegrino, P., Ferraro, R., Grody, N. C., and Meng, H.: A new snowfall detection algorithm over land using measurements from the Advanced Microwave Sounding Unit (AMSU), Geophys. Res. Lett., 30(14), 1756, doi:10.1029/2003GL017177, 2003.

Kummerow, C., Hong, Y., Olson, W. S., Yang, S., Adler, R. F., McCollum, J., Ferraro, R., Petty, G., Shin, D.-B., and Wilheit, T. T.: The evolution of the Goddard Profiling Algorithm (GPROF) for rainfall estimation from passive microwave sensors, J. Appl. Meteorol., 40, 1801-1820, 2001.

Liu, G.: Approximation of single scattering properties of ice and snow particles for high microwave frequencies, J. Atmos. Sci., 61, 2441-2456, 2004.

Meneghini, R. and Liao, L.: Comparisons of cross sections for melting hydrometeors as derived from dielectric mixing formulas and a numerical method, J. Appl. Meteorol., 35, 1658-1670, 1996.

Mugnai, A., Cooper, H. J., Smith, E. A., and Tripoli, G. J.: Simulation of microwave brightness temperatures of an evolving hail storm at SSM/I frequencies, B. Am. Meteorol. Soc., 71, 2-13, 1990.

Neshyba, S. P., Grenfell, T. C., and Warren, S. G.: Representation of a nonspherical ice particle by a collection of independent spheres for scattering and absorption of radiation: 2. Hexagonal columns and plates, J. Geophys. Res., 108, 4448-4465, 2003.

Olson, W., Bauer, P., Viltard, N. F., Johnson, D. E., Tao, W.-K., Meneghini, R., and Liao, L.: A melting-layer model for passive/active microwave remote sensing applications. Part I: Model formulation and comparison with observations, J. Appl. Meteorol., 40, 1145-1163, 2001.
Pruppacher, H. R. and Klett, J. D.: Microphysics of Cloud and Precipitation, Kluwer Academic, 914 pp., 1997.

Sanò, P., Casella, D., Formenton, M., Mugnai, A., Smith, E. A., Tripoli, G. J., and Yang, S.: Bayesian estimation of precipitating cloud parameters: Application to case studies of FLASH and RISKMED projects, Adv. Geosci., in preparation, 2008.

Skofronick-Jackson, G. M., Kim, M.-J., Weinman, J. A., and Chang, D.-E.: A physical model to determine snowfall over land by microwave radiometry, IEEE T. Geosci. Remote, 42, 10471058, 2004.

Tripoli, G. J.: A nonhydrostatic model designed to simulate scale interaction, Mon. Weather Rev., 120, 1342-1359, 1992.

Tripoli, G. J., Medaglia, C. M., Panegrossi, G., Dietrich, S., Mugnai, A., and Smith, E. A.: Modeling microphysical signatures of extreme events in the Western Mediterranean to provide a basis for diagnosing precipitation from space, in: Measuring Precipitation from Space: EURAINSAT and the Future, edited by: Levizzani, V., Bauer, P., and Turk, F. J., Springer, ISBN 1-40205834-9, 535-547, 2007.

van de Hulst, H. C.: Light Scattering Small Particles, Dover, New York, 470 pp., 1981.

Weinman, J. A. and Kim, M.-J.: A simple model of the millimeterwave scattering parameters of randomly oriented aggregates of finite cylindrical ice hydrometeors, J. Atmos. Sci., 64, 634-644, 2007.

Zhao, L. and Weng, F.: Retrieval of ice cloud parameters using the advanced microwave sounding unit, J. Appl. Meteorol., 41, 384 395, 2002. 